\section{KENTRON}

REVUE PLURIDISCIPLINAIR

DU MONDE ANTIQUE

\section{Kentron}

Revue pluridisciplinaire du monde antique

$31 \mid 2015$

Les Socratica de Xénophon

\title{
Ordre et progression des discours au chapitre IV du Banquet de Xénophon
}

\author{
Louis-André Dorion
}

\section{(2) OpenEdition}

Journals

Édition électronique

URL : http://journals.openedition.org/kentron/281

DOI : 10.4000/kentron.281

ISSN : 2264-1459

Éditeur

Presses universitaires de Caen

\section{Édition imprimée}

Date de publication : 1 novembre 2015

Pagination : 17-42

ISBN : 978-2-84133-747-7

ISSN : 0765-0590

\section{Référence électronique}

Louis-André Dorion, «Ordre et progression des discours au chapitre IV du Banquet de Xénophon », Kentron [En ligne], 31 | 2015, mis en ligne le 19 octobre 2016, consulté le 17 novembre 2020. URL http://journals.openedition.org/kentron/281 ; DOI : https://doi.org/10.4000/kentron.281

\section{(c) (i) (9)}

Kentron is licensed under a Creative Commons Attribution-NonCommercial-NoDerivatives 3.0 International License. 


\section{ORDRE ET PROGRESSION DES DISCOURS AU CHAPITRE IV DU BANQUET DE XÉNOPHON}

Au chapitre III du Banquet, Socrate rappelle à Callias qu'il aimerait bien le voir tenir sa promesse, en l'occurrence qu'il affiche son savoir ${ }^{1}$. Callias est disposé à s'exécuter, mais à la condition que les autres convives en fassent autant, c'est-à-dire qu'ils exposent chacun à tour de rôle ce qu'ils savent de bon ${ }^{2}$. Les discours de Callias et de ses hôtes, sur le thème de ce qui fait la fierté de chacun, occupent le chapitre IV, qui est le plus long chapitre du Banquet. L'ordre dans lequel se succèdent les discours, au chapitre IV du Banquet, n'est probablement pas fortuit, et j'aimerais vérifier, dans cette étude, l'hypothèse que cette succession obéit à un ordre croissant d'importance. Le texte du Banquet contient plusieurs indications qui permettent de départager les personnages entre ceux qui ne font pas partie de l'entourage de Socrate et ceux qui en font partie, et, parmi ces derniers, entre ceux qui prononcent un discours qui est endossé par Socrate et ceux qui tiennent au contraire des propos qui ne reçoivent pas l'approbation de Socrate. Soyons premièrement attentifs à ce que Xénophon rapporte dans les premiers paragraphes du Banquet:

Une fois les courses terminées, il [sc. Callias] se dirigea avec Autolycos et le père de celui-ci vers sa maison du Pirée; il était aussi accompagné de Nikératos. Ayant aperçu un groupe composé de Socrate, Critobule, Hermogène, Antisthène et Charmide, il chargea l'un de ses gens de conduire Autolycos et les autres, et s'avança vers Socrate et ses compagnons (I 2-4).

Les personnages mentionnés dans ce passage se partagent très nettement en deux groupes: d'une part, Callias, qui est l'hôte du banquet, son aimé Autolycos, le père de celui-ci et Nikératos, le fils de Nicias; d'autre part, Socrate et son entourage, à

1. III 3: «Pour ma part, dit Socrate, je serais très heureux d'obtenir de Callias l'accomplissement de sa promesse; car il a déclaré, n'est-il pas vrai? que si nous dînions ensemble, il nous prouverait

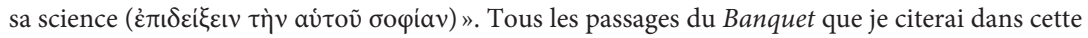
étude sont empruntés à la traduction de F. Ollier (1961).

2. III 3 : «Ainsi ferai-je, dit Callias, à condition que chacun de vous, sans nulle exception, nous expose

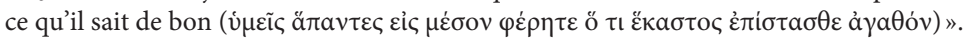


savoir Critobule, Charmide, Antisthène et, enfin, Hermogène. Les membres de l'entourage de Socrate ne sont pas tous, pour autant, des «socratiques", en ce sens que certains, parmi eux, tiennent des discours que Socrate ne pourrait pas prendre à son compte. Mais avant d'entreprendre l'analyse du chapitre IV et de partir à la recherche des éléments qui confirmeraient l'hypothèse suivant laquelle la succession des discours dudit chapitre marque une nette progression, il faut parler brièvement du chapitre III, qui expose d'une certaine façon le programme du chapitre IV. Les interlocuteurs sont en effet invités, au chapitre III, à dire brièvement ce qui les emplit de fierté, et ils auront ensuite l'occasion, au chapitre IV, d'exposer plus en détail l'objet de leur fierté. Or, comme on peut le constater d'après le tableau qui suit, la succession des interlocuteurs au chapitre III ne correspond pas tout à fait à celle du chapitre IV :

\begin{tabular}{|c|c|}
\hline Chapitre III & Chapitre IV \\
\hline Callias $(\$ 4)$ & Callias $(\$ 1-5)$ \\
\hline Nikératos $(\$ 5-6)$ & Nikératos $(\$ 6-9)$ \\
\hline Critobule $(\$ 7)$ & Critobule $(\$ 10-28)$ \\
\hline Antisthène $(\$ 8)$ & Charmide $(\$ 29-33)$ \\
\hline Charmide $(\$ 9)$ & Antisthène $(\$ 34-44)$ \\
\hline Socrate $(\$ 10)$ & Hermogène $(\$ 46-49)$ \\
\hline Philippe $(\$ 11)$ & Philippe $(\$ 50-51)$ \\
\hline Lycon $(\$ 12)$ & le Syracusain $(\$ 52-55)$ \\
\hline Autolycos $(\$ 12-13)$ & \\
\hline Hermogène $(\$ 14)$ & Socrate $(\$ 56-64)$ \\
\hline
\end{tabular}

Certains interlocuteurs du chapitre III (Lycon et son fils Autolycos) ne prennent pas la parole au chapitre IV, et, inversement, un interlocuteur du chapitre IV, le Syracusain, n'a pas pris la parole au chapitre III. Ces légères différences ne doivent cependant pas faire perdre de vue les éléments de continuité entre ces deux chapitres:

1) l'ordre des trois premiers discours (Callias, Nikératos, Critobule) est inchangé;

2) bien que l'ordre des interlocuteurs suivants soit bouleversé, on observe qu'à l'intérieur des couples Antisthène/ Charmide, d'une part, et Socrate / Hermogène, d'autre part, les personnages sont tout simplement permutés.

Les discours de Philippe (IV 50-51) et du Syracusain (IV 52-55) sont une espèce d'intermède ou de parenthèse avant le discours de Socrate (IV 56-64), de sorte qu'ils ne font pas vraiment partie de la gradation croissante des discours. Quelles que soient la portée et la signification des différences entre les chapitres III et IV, c'est surtout la succession des discours au chapitre IV qui importe, car le chapitre III n'est guère plus qu'une anticipation du chapitre IV. Ce sont, en effet, les discours 
du chapitre IV qui sont les plus développés et qui permettent le mieux de saisir la progression entre les différents interlocuteurs.

On peut établir une première division entre Callias et Nikératos d'une part, Socrate et les membres de son entourage d'autre part. Mais on peut également procéder à une seconde division, au sein de l'entourage de Socrate, entre ceux qui tiennent des propos non socratiques (Critobule, Charmide) et ceux qui expriment un point de vue qui est endossé par Socrate (Antisthène, Hermogène). On aurait ainsi une progression en quatre temps: 1) les discours des personnages extérieurs au cercle socratique et qui ne sont pas socratiques sous le rapport de leur contenu et des idées exprimées (Callias et Nikératos); 2) les discours prononcés par des compagnons de Socrate, mais qui ne peuvent pas être endossés par ce dernier (Critobule et Charmide) ; 3 ) les discours prononcés par des compagnons de Socrate et qui sont fidèles à son enseignement (Antisthène et Hermogène); 4) enfin, en guise de couronnement, le discours de Socrate lui-même. Les trois derniers discours se qualifient du titre de «socratiques» (Antisthène, Hermogène, Socrate) dans la mesure où ils exposent, à la différence des quatre premiers discours (Callias, Nikératos, Critobule, Charmide), des idées et des positions que Xénophon prête à Socrate dans ses autres écrits. On peut, en effet, montrer que les discours d'Antisthène, d'Hermogène et, bien sûr, de Socrate lui-même sont corroborés par des idées exprimées par Socrate dans les autres écrits socratiques de Xénophon.

Le partage que j'effectue entre deux groupes d'interlocuteurs me semble confirmé, à une exception près, par un élément narratif qui peut sembler anodin, mais qui a probablement une certaine importance. Tous les interlocuteurs du premier groupe s'attribuent à eux-mêmes la parole, sans qu'on les invite à parler - Callias ${ }^{3}$, Nikératos ${ }^{4}$, Critobule ${ }^{5}$-, alors que les interlocuteurs du deuxième groupe attendent qu'on leur donne la parole: Charmide ${ }^{6}$, Antisthène ${ }^{7}$, Hermogène ${ }^{8}$, Socrate ${ }^{9}$.

3. IV 1: «Je vous prie de m'écouter le premier, dit Callias».

4. IV 6: «Ainsi finit la discussion. Nikératos prit alors la parole: "Apprenez de moi à mon tour, je vous prie, en quoi vous deviendrez meilleurs, si vous me fréquentez" ".

5. IV 10: «C'est à peu près ainsi que l'on en termina avec ce sujet. “Ả mon tour donc, fit alors Critobule; je vais expliquer pourquoi je suis fier de ma beauté" ».

6. IV 29: «Voilà comment ils mêlaient le plaisant au sérieux. Alors Callias: "À ton tour, dit-il, Charmide, d'expliquer pourquoi tu es fier de ta pauvreté" ".

7. IV 34: «Allons, dit Socrate, à toi maintenant de nous dire, Antisthène, comment il se fait qu'étant si à court de biens tu sois fier de ta richesse».

8. IV 46: «Quelqu'un dit ensuite: “À toi, Hermogène; fais-nous connaître qui sont tes amis, et montre-nous toute l'étendue de leur pouvoir et de leur sollicitude pour ta personne, afin qu'elle paraisse justifiée, la fierté que tu éprouves à leur sujet" ».

9. IV 56: «Bon, dit Callias; quant à toi, Socrate, de quelle façon peux-tu soutenir que tu as le droit d'être fier du métier si décrié que tu as nommé?». 
Critobule serait donc la seule exception à cette «règle». L'empressement de Callias, Nikératos et Critobule à prendre la parole, sans même qu'on les ait invités à le faire, rappelle le semblable empressement du Vice, dans l'apologue d'Héraklès à la croisée des chemins, à prendre la parole avant la Vertu ${ }^{10}$. Nous aurions ainsi une première indication que les premiers à prendre la parole sont aussi les plus éloignés de la vertu (Socrate).

Procédons maintenant à l'examen de chacun des sept discours exposés au chapitre IV, afin de vérifier s'il est exact que la succession de ces discours marque une progression qui va dans le sens d'un rapprochement avec les positions défendues par Socrate.

\section{Callias (IV 1-5)}

Callias est pressé de parler en premier $\left(\$_{1}\right)$, ce qui montre bien à quel point il est vaniteux ${ }^{11}$ et à quel point il ne se doute pas que cet empressement se retournera contre lui. Il se déclare fier de pouvoir rendre les hommes meilleurs par son argent. L'ambition de Callias est en un sens la même que celle de Socrate - rendre les hommes meilleurs -, mais le moyen de réaliser cette ambition, l'argent, n'a rien de socratique. L'objection qu'Antisthène formule est fatale à la prétention de Callias. Cette objection insiste sur l'absence de réciprocité: Callias prétend rendre les autres hommes justes en leur donnant de l'argent, mais, reconnait-il, ces hommes ne le «payent» pas en retour, de sorte qu'ils se montrent ainsi ingrats envers leur bienfaiteur. Or, l'ingratitude est pour Xénophon une forme très grave d'injustice ${ }^{12}$, voire l'injustice à l'état pur. La prétention de Callias est démentie par les faits: il prétend rendre les hommes plus justes, mais ceux-ci, en ne lui témoignant pas leur gratitude, se montrent injustes à son endroit. Contrairement à ce que soutient Callias, la véritable condition de la justice n'est pas l'argent, mais la maitrise de soi (enkrateia $)^{13}$. Or, l'enkrateia est une force qui réside dans l'âme, ce qui va nettement dans le sens de la position défendue par Antisthène. Même si l'on peut avoir l'impression que Socrate vole au secours de Callias $\left(\$_{5}\right)$, il ne fait aucun doute, à la lumière d'un passage des Mémorables, qu'il se range aux côtés d'Antisthène ${ }^{14}$ et qu'il désavoue la position de Callias:

10. Cf. Mém. II 1, 23: "Quand elles se furent approchées d'Héraklès, celle dont nous avons parlé en premier [sc. la Vertu] s'avança sans presser le pas, alors que l'autre [sc. le Vice], qui voulait la devancer, se précipita sur Héraklès et lui dit $[\ldots] »$.

11. Cf. Ollier 1961, 51, n. 1; Carrière 1998, 248-249.

12. Cf. Mém. II 2, 1-3; IV 4, 24; Agés. IV 2; Cyr. I 2, 7 ; Hell. IV 1, 33.

13. Cf. Apol. 16; Mém. I 2, 1-8; IV 2, 38; Dorion 2013, XxI-XXII.

14. Cf. Huss 1999, 204. 
Par Héra, répondit Socrate, je t’admire [sc. Euthydème] de ne pas avoir préféré les trésors d'argent et d'or aux trésors du savoir; car à l'évidence tu considères que

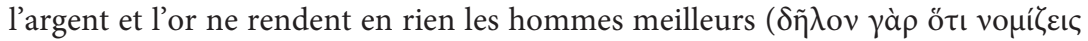

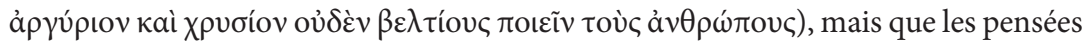
des savants enrichissent de vertu ceux qui les détiennent ( $\tau$ àc $\delta \grave{\varepsilon} \tau \tilde{\omega} v \sigma o \varphi \tilde{\omega} v$ ảv $\delta \rho \tilde{\omega} v$

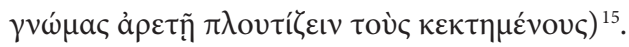

Cette affirmation de Socrate peut être lue comme un désaveu de ce qui fait la fierté de Callias $(\$ 1)$, à savoir la prétention à rendre les hommes plus justes en leur donnant de l'argent. Socrate ne conteste pas ouvertement cette position de Callias, sans doute par crainte de froisser son hôte, et Xénophon confie à Antisthène la tâche ingrate de rabrouer Callias et de le réfuter en lui faisant comprendre que ce n'est pas par des dons en argent que l'on rend les hommes plus vertueux (\$1-4).

\section{Nikératos (IV 5-9)}

Nikératos n'est pas un socratique. Son père, le célèbre Nicias, aurait bien aimé que Socrate acceptât de s'occuper de son fils et l'admît dans son entourage, mais Socrate a refusé de le prendre sous son aile ${ }^{16}$, sans doute parce que Nikératos n'avait pas les qualités intellectuelles requises.

Nikératos est fier de sa connaissance d'Homère. Il soutient qu'il peut être utile à tous les hommes dans la mesure où il connaît Homère par cœur; comme Homère traite de toutes les activités humaines, et que Nikératos connaît Homère par cœur, il pourra instruire les hommes en toute chose et les rendre meilleurs. La position défendue par Nikératos correspond à celle qui est attaquée par Socrate dans I'Ion ${ }^{17}$ : les rhapsodes connaissent certes par cœur les poèmes d'Homère, mais détiennent-ils pour autant une compétence en chacune des techniques mises en scène par Homère? Or, Nikératos s'imagine qu'il est savant dans tous les domaines d'activités que décrit Homère, si bien que les hommes qui désirent devenir stratèges ou administrateurs n'ont qu'à cultiver sa société:

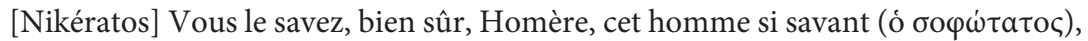
a traité dans ses vers de presque toutes les activités humaines. C'est pourquoi quiconque parmi vous voudra devenir capable de bien administrer sa maison

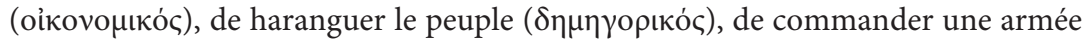

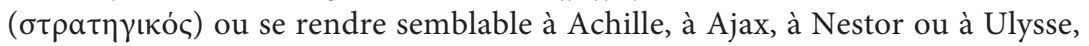

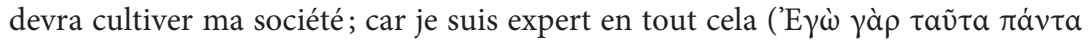

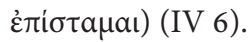

15. Mém. IV 2, 9 (trad. Dorion 2011b).

16. Cf. Lachès $200 \mathrm{c}-\mathrm{d}$.

17. Cf. surtout $536 \mathrm{~d}-542 \mathrm{~b}$. 
Or, ce n'est pas ainsi qu'une compétence se transmet ou s'acquiert. Xénophon affirme à de nombreuses reprises que l'acquisition d'une compétence donnée suppose la fréquentation d'un maître compétent en ce domaine ${ }^{18}$, et non pas la fréquentation d'un rhapsode. Xénophon lui-même n'aurait pas composé l'Économique, s'il avait cru qu'il suffit de fréquenter un rhapsode pour apprendre à administrer son domaine; lorsque Critobule le consulte parce qu'il a besoin de conseils sur la meilleure façon d'administrer un domaine, Socrate ne lui recommande pas de fréquenter un rhapsode, mais il lui rapporte plutôt un long entretien qu'il a eu avec un homme qui a la réputation d'être un administrateur prospère (Ischomaque). De même, lorsqu'il apprend qu'un de ses compagnons aspire à devenir stratège, Socrate ne lui conseille pas de cultiver la société d'un rhapsode, mais il l'envoie plutôt suivre les leçons d'un homme (Dionysodore) qui prétend être un maître de stratégie ${ }^{19}$. Si cette critique des prétentions de Nikératos suppose un détour par l'Économique et les Mémorables, il en est une autre qu'Antisthène adresse directement à Nikératos:

[Antisthène] - Connais-tu donc une engeance plus sotte ( $\left.\lambda_{\imath} \theta_{\imath} \iota \dot{\omega} \tau \varepsilon \rho o v\right)$ que celle des rhapsodes? - Non, par Zeus, répondit Nikératos, non vraiment je ne le crois pas. - Il est clair, en effet, dit Socrate, qu'ils ne connaissent pas le sens caché des vers

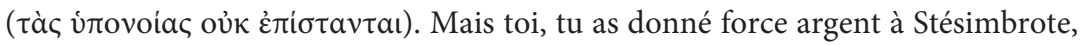
à Anaximandre et à quantité d'autres, si bien que rien ne t'échappe de ce qu'ils contiennent de précieux ${ }^{20}$.

Nikératos a tort d'être fier de connaitre Homère par cœur, car ce n'est rien de plus qu'une connaissance de perroquet: non seulement il n'a pas pour autant la connaissance de tous les sujets qui sont traités par Homère, mais il n'a pas non plus accès au sens caché des vers. Et de même que Callias a tort de croire qu'il peut rendre les hommes justes en leur donnant de l'argent, de même Nikératos se trompe en s'imaginant qu'il suffit de donner de l'argent à des professeurs pour avoir accès au sens caché des vers. Bref, Nikératos ne partage rien en commun avec Socrate: il ne fait pas partie de son entourage et il défend une position qui est ouvertement désavouée par Socrate.

18. Cf. Mém. I 6, 14; III 1, 1-3; IV 2, 1-10; IV 4, 5; IV 7, 1.

19. Cf. Mém. III 1. Peu importe que Dionysodore se révèle un maître incompétent. Son incompétence ne change rien au fait que la stratégie, comme n'importe quelle autre discipline, doit s'apprendre auprès d'un maître compétent.

20. Banq. III 6. La sottise des rhapsodes est à nouveau dénoncée dans les Mémorables: «[Socrate] Eh bien, ne veux-tu pas devenir rhapsode? Car on dit aussi que tu possèdes toute la poésie d'Homère. - [Euthydème] Non, par Zeus, je ne le veux pas, répondit-il. Je sais en effet que les rhapsodes savent

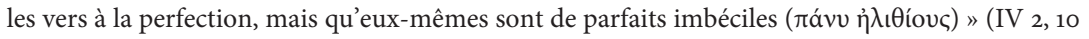
[trad. Dorion 2011b]). 


\section{Critobule (IV 10-28)}

Alors que Nicias n'a pas réussi à convaincre Socrate de s'occuper de son fils Nikératos $^{21}$, Criton n'a eu, semble-t-il, aucune peine à convaincre son bon ami Socrate de veiller sur son fils Critobule (cf. IV 24). Celui-ci est un personnage mineur dans les dialogues de Platon ${ }^{22}$, mais c'est à coup sûr un personnage central dans les écrits socratiques de Xénophon ${ }^{23}$. Il est, en effet, l'interlocuteur de Socrate dans l'Économique et dans l'un des plus longs entretiens rapportés dans les Mémorables, à savoir celui qui porte sur l'amitié au chapitre 6 du livre II. Il ne fait donc aucun doute que Critobule fait partie de l'entourage de Socrate; mais il n'est pas pour autant un "socratique», dans la mesure où il incarne, dans les dialogues socratiques de Xénophon, la figure du jeune homme dépourvu d'enkrateia, qui court à sa perte et à sa ruine en raison de sa passion pour les beaux garçons ${ }^{24}$.

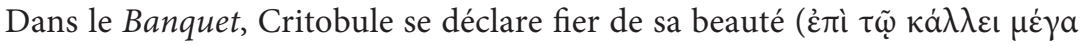
$\varphi \rho o v \tilde{\omega}$, IV 10). Son discours contient de nombreuses affirmations qui paraissent non socratiques pour autant qu'elles sont contraires à des positions défendues par Socrate. Parmi les nombreuses divergences que l'on peut relever entre les affirmations de Critobule et les positions de Socrate ${ }^{25}$, celles-ci retiennent surtout notre attention:

a) Critobule affirme à de nombreuses reprises qu'il est beau ${ }^{26}$ et qu'il ne considère que les beaux garçons ${ }^{27}$. Or Socrate ne recherche pas la beauté physique, mais celle de l'âme ${ }^{28}$.

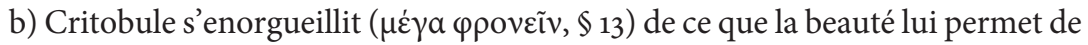

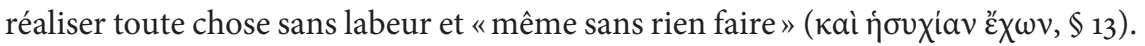
Le point de vue exprimé par Critobule rejoint celui du Vice en Mém. II 1, lorsqu’il promet à Héraklès qu'il jouira de tous les plaisirs sans effort et sans travail ${ }^{29}$. Or, pour Socrate et Xénophon, tous les biens doivent au contraire s'obtenir au prix

21. Cf. Lachès 2ooc-d.

22. Critobule est parfois mentionné dans les dialogues de Platon (cf. Apol. 33e, 38b, Euthyd. 306d-e, Phédon 59b), mais il ne s'entretient jamais avec Socrate.

23. Voir, dans ce présent numéro, l'article de Marie-Pierre Noël, «Critobule dans les écrits socratiques de Xénophon: le portrait d'un mauvais élève», p. 43-58.

24. Cf. Mém. I 3, 8-13; II 6, 31-32; Écon. II 7; Dorion 2011a, 34 n. 6.

25. Voir, en Annexes à cette étude, le Tableau des correspondances et des divergences entre Socrate et ses compagnons.

26. Cf. IV 10; IV 11; IV 13; IV 15; IV 16; IV 19; etc.

27. Cf. IV 11; IV 12; Mém. II 6, 30-33.

28. Cf. Mém. IV 1, 2: «Il se disait souvent amoureux de quelqu'un, mais, à l'évidence, il ne recherchait pas ceux que la nature avait heureusement dotés de corps attrayants, mais ceux que la nature avait heureusement dotés d'âmes portées à la vertu ».

29. Cf. Mém. II 1, 23-25. 
du travail et de l'effort (ponos) ${ }^{30}$. Certes, Socrate ne conteste pas ouvertement la position de Critobule, mais son silence ne doit pas être interprété comme un signe d'approbation. Au chapitre VIII, dans son long discours sur l'amour, Socrate insiste à trois reprises sur l'importance de l'effort et du labeur (ponos) ${ }^{31}$.

c) Critobule affirme qu'il aurait plus de plaisir à être esclave qu'à être libre, pourvu qu'il ait Clinias pour maître $(\$ 14)$. Or, Socrate condamne sans appel le choix de celui qui, par amour, préfère l'esclavage à la liberté ${ }^{32}$.

d) Critobule considère que "c'est folie que de ne pas choisir de beaux hommes

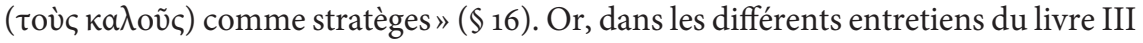
des Mémorables ${ }^{33}$ où Socrate traite des vertus, des qualités et des compétences que doit détenir le stratège, il n'est jamais question de sa beauté physique.

e) Au $\$ 18$, Critobule s'enorgueillit de ce que sa beauté lui confère cette supériorité sur Socrate:

Et s'il est doux d'obtenir des gens qu'ils se rendent volontiers à nos désirs, je suis certain qu'en ce moment j'aurais plus vite fait de persuader (åv $\dot{\varepsilon} \gamma \grave{\omega}[\ldots] \pi \varepsilon \dot{i} \sigma \alpha \mu \mu)$, même sans dire mot, le jeune garçon et la jeune fille que voici de m'embrasser ( $\varphi \iota \lambda \tilde{\eta} \sigma \alpha \iota)$ que toi, Socrate, même si tu y employais toute ton éloquence ( $\varepsilon \grave{i}$ kaì

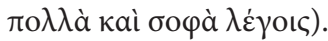

Cette prétention de Critobule est contredite par deux textes précis. Premièrement, alors que la courtisane Théodote avait la réputation d'être d'une beauté qui dépassait

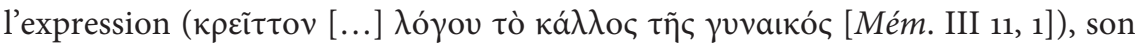
entretien avec Socrate, au cours duquel elle est complètement séduite par ce dernier, démontre à l'évidence que le pouvoir de séduction de Socrate, qui repose entièrement sur la parole, est de loin supérieur à celui de Théodote, de sorte qu'il est faux

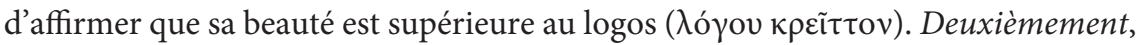
en affirmant que sa beauté physique lui permettrait de convaincre le jeune garçon et la jeune fille de l'embrasser, Critobule tombe à l'avance sous le coup du blâme que Socrate prononcera dans son discours du chapitre VIII:

30. Cf. Mém. I 2, 1-2; II 1, 3; II 1, 15; II 1, 18-20; II 1, 28; Écon. V 4; Cyr. VI 2, 4; VII 5, 74; VII 5, 80; VIII 6, 12 ; etc.

31. Cf. VIII $32 ; 37$ et 40.

32. Cf. Mém. I 3, 11: «Malheureux, répondit Socrate, as-tu une idée de ce qui t'arrivera après avoir embrassé un joli garçon? Ne sais-tu pas que de libre tu deviendras aussitôt esclave ( ${ }^{\sim} A \rho$ ' oủk ầ $v$

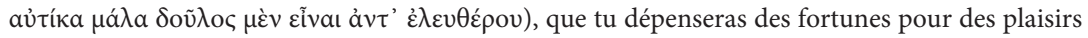
funestes, que tu seras privé du loisir de t'occuper de ce qui est beau et bon, et que tu seras contraint de t'appliquer à des choses auxquelles personne, pas même un fou, ne s'appliquerait? (trad. Dorion 2000)

33. Cf. Mém. III 1; III 2; III 4; III 5. 


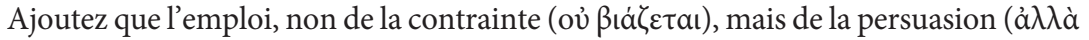

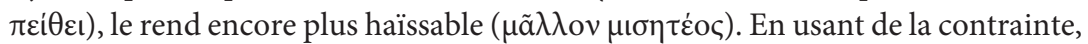
en effet, on ne fait que montrer sa perversité, mais en employant la persuasion on

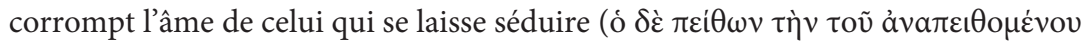

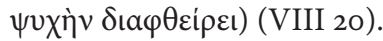

Or, Critobule est justement prêt à user du pouvoir de persuasion que lui confère sa beauté pour obtenir des baisers du jeune garçon ou de la jeune fille. Certes, Socrate est lui aussi un redoutable séducteur qui opère par la persuasion, et non par la contrainte, mais la séduction qu'il exerce ne vise jamais à obtenir des faveurs sexuelles.

f) $\mathrm{Au} \S 23$, Hermogène fait cette observation à Socrate à propos de Critobule: «Ah! pour moi, Socrate, je ne trouve pas digne de toi que tu t'inquiètes si peu de

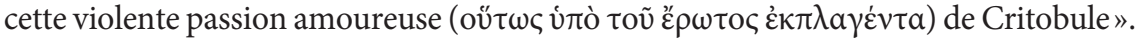
L'effet de la passion amoureuse - physique - qui s'est emparée de Critobule est

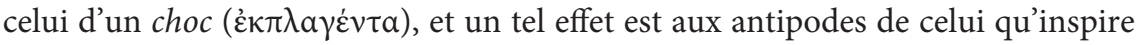
l'«amour modéré», celui-là même qu'inspire la vue d'Autolycos et que Xénophon décrit en ces termes au début du Banquet:

Tous ceux qui sont possédés par un dieu valent, semble-t-il, la peine d'être vus; mais alors que la possession d'autres divinités entraîne des regards terribles, une voix effrayante et des gestes violents, ceux qui sont possédés par le chaste Amour

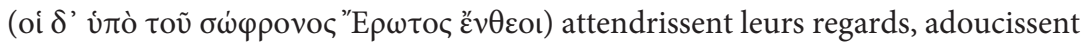
leur voix et accroissent la noblesse de leurs attitudes (I 10).

Or, le choc que provoque chez Critobule la passion amoureuse, de nature purement physique, ne peut pas être approuvé par Socrate, car c'est précisément le genre de choc qui a pour effet, chez celui qui en est la victime, de brouiller la perception du bien et du mal et de provoquer de mauvais choix ${ }^{34}$.

La réfutation de Critobule ne se situe pas dans l'intervention que Socrate fait à l'invitation d'Hermogène (IV 23), qui s'étonne de ce que Socrate semble faire si peu de cas de la passion de Critobule pour Clinias. De fait, Socrate ne semble pas s'en alarmer. Est-ce à dire qu'il approuve le discours et le comportement de Critobule? Certainement pas. S'il ne le réfute pas sur-le-champ, c'est probablement parce qu'il préfère attendre de prononcer son long discours sur l'amour (ch. VIII). Le cas de Critobule est digne d'intérêt en ce qu'il montre les limites de l'enseignement dispensé par Socrate; autrement dit, Socrate comptait manifestement dans son entourage des disciples qui résistaient à son enseignement et ainsi en révélaient les limites.

34. Cf. Mém. IV 5, 6. 


\section{Charmide (IV 29-33)}

Dans un entretien des Mémorables (III 7), Socrate encourage Charmide à vaincre ses réticences à faire de la politique et à plonger dans l'arène politique, ce qui ne laisse pas d'être troublant, si l'on songe à la participation de Charmide à la tyrannie des Trente. L'identification du Charmide du Banquet au Charmide des Mémorables n'est pas d'emblée évidente, car on voit mal comment Socrate pourrait encourager le Charmide du Banquet à faire de la politique. Aucune des qualités que Socrate reconnaît à Charmide, dans les Mémorables, n'est en effet présente chez le Charmide du Banquet. En tout état de cause, l'appartenance de Charmide au cercle des intimes de Socrate dans le Banquet est confirmée par deux passages (I 3-4; IV 32), et cela suffit probablement à établir que nous ne sommes pas en présence de deux personnages homonymes, soit le Charmide du Banquet et celui des Mémorables (III 7) ${ }^{35}$.

Charmide se déclare fier de sa pauvreté (\$29), et il tient des propos que Socrate pourrait difficilement approuver:

a) Dans l'Économique (XI 3), Socrate déclare que le reproche le plus insensé qu'on lui ait jamais adressé est de le traiter de pauvre ${ }^{36}$. Malgré les apparences, Socrate n'est pas pauvre; certes, il possède peu de choses, mais il n'en est pas moins riche, ainsi qu'il le déclare lui-même ${ }^{37}$, car la véritable richesse consiste, non pas à posséder beaucoup de biens, mais à posséder plus que ce dont on a besoin. Socrate voit dans la pauvreté non seulement la conséquence d'un manque d'enkrateia, mais aussi le signe d'une inaptitude à faire de la politique dans la mesure où la maitrise de soi est la condition du gouvernement d'autrui ${ }^{38}$. Socrate ne pourrait donc jamais prétendre qu'il est fier de sa pauvreté.

b) Au $\$ 30$, Charmide fait la confession suivante: «je faisais la cour aux syco-

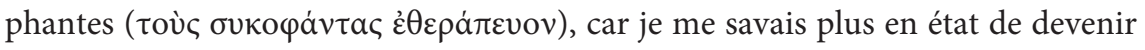

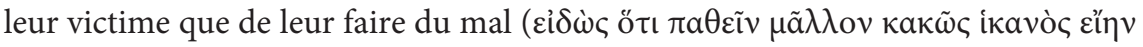

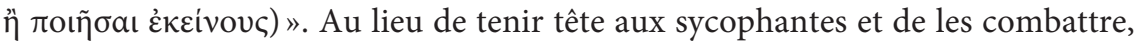
ainsi que le fait le riche Criton avec l'aide et les conseils de Socrate ${ }^{39}$, Charmide les «soigne» ( $\dot{\varepsilon} \theta \varepsilon \rho \alpha \dot{\pi} \varepsilon v o v)$, c'est-à-dire qu'il les courtise, de crainte d'en être la victime. Au vu du chapitre des Mémorables (II 9) où Socrate met Criton en relation avec

35. Sur le personnage de Charmide dans les dialogues socratiques de Platon et de Xénophon, cf. Nails 2002, 90-94, s.v. "Charmides of Athens, son of Glaucon III ».

36. "Allons donc, ai-je répondu, comment aurais-je le droit de redresser un homme "bien", un homme accompli, moi qui passe pour un moulin à paroles, un arpenteur de nuées, moi que - grief

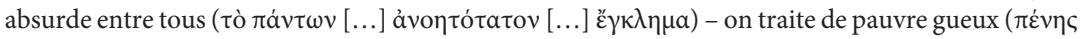

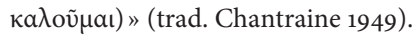

37. Cf. Écon. II 2.

38. Cf. Mém. I 5, 1; II 1; IV 5, 12.

39. Cf. Mém. II 9. 
un homme qui le débarrassera des sycophantes qui le harcèlent, la complaisance de Charmide à l'endroit des sycophantes ne serait certainement pas approuvée par Socrate. De plus, en admettant qu'il est plus en état de souffrir du mal de la part des sycophantes que de leur en infliger par lui-même, Charmide reconnaît par le fait même qu'il ne satisfait pas à une exigence de l'homme de bien, dont la vertu, selon Socrate, est précisément de l'emporter sur ses ennemis en mauvais traitements ${ }^{40}$.

c) Lorsqu'il était riche, Charmide était en butte aux menaces et il se félicite, maintenant qu'il est devenu pauvre, de pouvoir à son tour menacer ses concitoyens $(\$ 31)$. Charmide ne précise pas la nature des menaces dont il était l'objet et qu’il profère à son tour, mais l'allusion aux sycophantes $(\$ 31)$ donne à penser qu'il s'est rangé dans leur camp. C'est probablement parce qu'il n’hésite pas à faire le sycophante que les riches se lèvent de leurs sièges à son passage et qu'ils s'écartent devant ses pas $(\$ 31)$. Xénophon rapporte que Socrate était sévère à l'endroit des riches qui se montraient arrogants et inutiles ${ }^{41}$, mais il ne faut pas en conclure que l'attitude de Socrate à l'endroit des riches rejoint celle de Charmide. Ce dernier menace les riches du seul fait qu'ils sont riches, alors que la sévérité de Socrate à l'endroit des riches est sélective, en ce sens qu'elle vise ceux d'entre eux qui refusent de prêter main-forte à la cité, à l'armée ou au peuple.

Ni Antisthène ni Socrate n'interviennent pour interroger Charmide ou pour lui faire des objections. Leur silence ne vaut pas pour autant approbation, car le discours qui suit, celui d'Antisthène, prend le contre-pied de celui prononcé par Charmide.

\section{Antisthène (IV 34-45)}

À l'instar de Critobule, Antisthène est beaucoup plus présent dans les écrits socratiques de Xénophon qu'il ne l'est dans les dialogues de Platon. Il est l'interlocuteur de Socrate dans un entretien des Mémorables (II 5), et Xénophon le présente comme un intime de Socrate ${ }^{42}$.

Les discours de Charmide et d'Antisthène se répondent l'un à l'autre, comme c'est souvent le cas, dans les Mémorables, entre des entretiens distincts. Or, lorsqu'on est en présence d'entretiens dont l'un est l'envers symétrique de l'autre, il y en a toujours un qui représente la version positive d'un thème dont l'autre entretien est la version négative. En voici trois exemples:

40. Cf. Mém. II 6, 35 .

41. «Il disait plutôt que ceux qui ne sont utiles ni en parole ni en acte, et qui sont incapables, en cas de besoin, de prêter main-forte à l'armée, à la cité ou au peuple, doivent à tout prix être réprimés

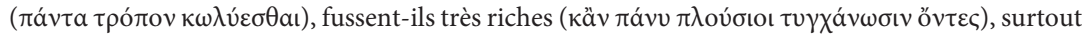
s'ils sont arrogants en plus d'être inutiles» (Mém. I 2, 59 [trad. Dorion 2000]).

42. Cf. Mém. III 11, 17; Banq. IV 44; VIII 4-6. 
a) Alors que Glaucon, le frère de Platon, est impatient de faire de la politique, et que Socrate doit intervenir pour lui démontrer qu'il n'a pas encore les compétences nécessaires pour entreprendre une carrière politique (Mém. III 6), Charmide est au contraire réticent à faire de la politique, et Socrate doit intervenir pour le convaincre qu'il détient la capacité (dunamis) appropriée pour s'occuper activement des affaires de la cité (Mém. III 7).

b) Alors qu'Alcibiade ne se satisfait pas de la définition de la loi que lui fournit Périclès, à tel point qu'il critique et réfute Périclès avec insolence ${ }^{43}$, Socrate, au livre IV, propose et défend une définition de la loi qui est identique à celle que Périclès avait soumise à Alcibiade ${ }^{44}$.

c) Alors que la façon dont Critias cherche à séduire Euthydème est indigne d'un homme libre - Socrate lui reproche, en effet, de «quêter» les faveurs d'Euthydème et de se frotter contre lui comme les porcs contre les pierres ${ }^{45}$-, Socrate parvient à séduire Euthydème en usant uniquement des ressources de la parole (Mém. IV 2).

Ces exemples m'incitent à considérer que le discours de Charmide est la version négative, c'est-à-dire non socratique, de ce dont le discours d'Antisthène représente la version positive. Antisthène, qui est fier de sa richesse (IV 34), prononce en effet un discours qui marque un net progrès par rapport à celui de Charmide. Tout au début de son discours, Antisthène affirme que «les hommes détiennent la richesse

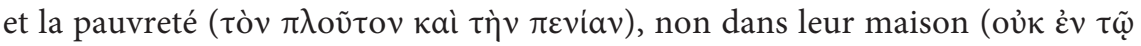

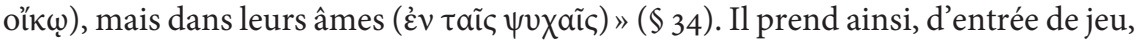
le contre-pied de la position de Charmide. La pauvreté de Charmide est, en effet, purement matérielle, en ce sens qu'elle consiste en la perte de biens extérieurs, et ce qu'il dit de son espoir d'acquérir quelque chose (\$32-33) montre bien qu'il n’y a rien de changé en son âme, c'est-à-dire qu'il ne se satisfait pas de sa pauvreté et qu'il n'a pas renoncé à redevenir riche. Charmide n'a donc pas compris une chose essentielle, à savoir que la pauvreté et la richesse ne se mesurent pas à la quantité de choses que l'on possède, mais à la capacité de chacun à satisfaire à ses besoins, de sorte que celui qui réduit ses besoins au strict minimum, comme c'est le cas de Socrate et d'Antisthène, peut être riche avec peu de biens.

Les recoupements entre les affirmations d'Antisthène et les positions défendues par Socrate, dans les Mémorables, sont nombreux ${ }^{46}$, et ils attestent que la position d'Antisthène est authentiquement socratique. Je me contenterai ici de souligner un seul de ces recoupements. Au $\$ 36$, Antisthène exprime une idée chère à Socrate et

\footnotetext{
43. Cf. Mém. I 2, 40-46.

44. Cf. IV $4,13 s q$.

45. Cf. Mém. I 2, 30.

46. Voir, en Annexes à cette étude, le Tableau des correspondances et des divergences entre Socrate et ses compagnons.
} 
à Xénophon: l'absence de modération conduit à l'injustice. Celui qui n'a pas appris à refréner ses appétits et à limiter ses désirs ne se satisfait jamais de ce qu'il possède, et il est ainsi conduit à convoiter le bien d'autrui, d'où les actes injustes, aussi bien individuels (vols) que collectifs (guerres).

Plutôt que de reprendre un à un les éléments du discours d'Antisthène et de montrer pour chacun d'eux les liens étroits qui les unissent aux éléments correspondants de la conception socratique de l'autarcie, je préfère m'attarder sur certaines différences qui m’apparaissent révélatrices, en ceci qu'elles suggèrent une certaine dissymétrie entre, non pas les conceptions respectives que Socrate et Antisthène se font de l'autarcie - c'est fondamentalement la même -, mais plutôt entre leur degré respectif d'autosuffisance. Une lecture attentive des Mémorables et du Banquet révèle que Xénophon s'est appliqué à suggérer qu'Antisthène était moins avancé que Socrate sur la voie de l'autarcie. Il y a, en effet, quelques indices qui trahissent ce «retard» d'Antisthène:

a) Antisthène reconnaît ouvertement qu'il doit à Socrate la forme de richesse qui consiste à n'avoir besoin de rien (IV 43). Rappelons que Socrate s'efforce de rendre ses disciples autarciques ${ }^{47}$; or, comme Antisthène est l'un des plus proches disciples de Socrate, il est tout à fait naturel qu'il reconnaisse sa dette à l'endroit de son maître.

b) Au $\$ 40$, Antisthène affirme ce qui suit: «Mais ce que je compte de plus précieux dans ma richesse, c'est que, si l'on m'enlevait même ce que j'ai à présent, je ne vois pas d'occupation si humble qui ne puisse me procurer de quoi manger à

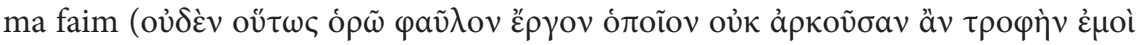
$\pi \alpha \rho \varepsilon ́ \chi 0 ı) »$. On trouve dans les Mémorables un parallèle frappant, où Xénophon s'exprime presque dans les mêmes termes à propos de Socrate:

Il avait plié son âme et son corps à un régime tel qu'il permettait à celui qui l'adoptait, sauf extraordinaire, de vivre en confiance et en sécurité, et de n'être jamais à court de ressources pour subvenir à une dépense si modeste. Il était à ce point frugal que je me demande si tout travail, même minime, ne procure pas davantage que ce

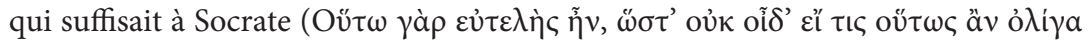

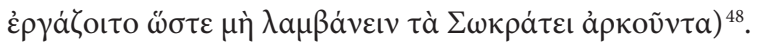

Le parallèle entre les deux textes est frappant, mais il ne faut cependant pas négliger cette différence: Antisthène se contente certes de peu, mais il est moins autarcique que Socrate, puisqu'il avoue lui-même qu'il aurait néanmoins besoin d'un « humble

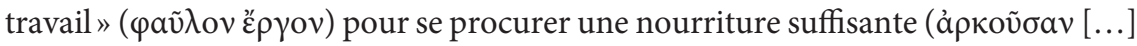

47. Cf. Mém. IV 7, 1.

48. Mém. I 3, 5 (trad. Dorion 2000). 


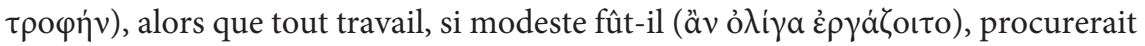
inévitablement à Socrate plus qu'il ne lui en faut, de sorte qu'il n'a pas besoin de travailler.

c) Antisthène reconnaît, au $\$ 38$, qu'il dort si bien, quand il est à la maison, que

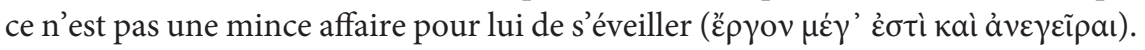
Or, c'est là une «faiblesse» que Xénophon n'attribue jamais à Socrate. Comme Socrate est l'homme qui fait preuve de la plus grande maitrise de soi à l'endroit des plaisirs, et que le sommeil compte au nombre des plaisirs à l'endroit desquels s'exerce l'enkrateia ${ }^{49}$, Socrate maîtrise parfaitement le sommeil, à tel point que Xénophon ne rapporte jamais qu'il dort, et encore moins qu'il prend du plaisir à dormir ou qu'il a de la peine à s'éveiller.

d) La principale différence entre Socrate et Antisthène concerne la dimension sexuelle de leur autarcie respective. Alors que Xénophon s'applique à montrer que Socrate s'abstenait d'avoir des relations sexuelles avec les garçons et les courtisanes, même si par ailleurs il ne désapprouvait pas les hommes qui faisaient appel aux services de prostituées ${ }^{50}$, Antisthène avoue sans détour et sans vergogne non seulement qu'il soulage son désir avec les "premières venues", mais encore qu'il éprouve à leur commerce plus de plaisir qu'il n'est utile:

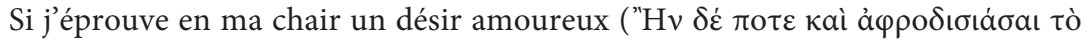

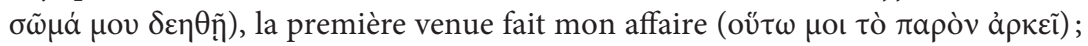
aussi les femmes dont je m'approche me comblent-elles de caresses, parce que nul autre ne consent à aller avec elles. Et toutes ces jouissances me semblent d'un tel agrément que je souhaiterais non pas trouver plus de plaisir en chacune d'elles, mais au contraire en éprouver moins; tant quelques-unes me paraissent plus agréables

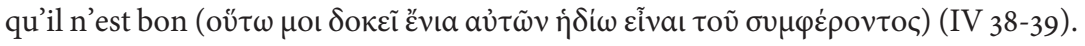

Il me parait très éclairant de rapprocher cet «aveu» d'un passage des Mémorables qui expose la "diète" sexuelle de Socrate et les conseils qu'il prodiguait en matière de régime sexuel:

Il croyait que ceux qui sont susceptibles de succomber aux plaisirs d'Aphrodite

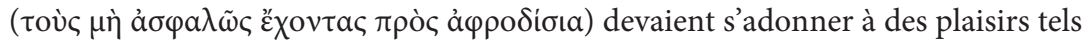
que l'âme ne les accepterait pas en l'absence d'un besoin impérieux du corps ( $\pi$ ávv

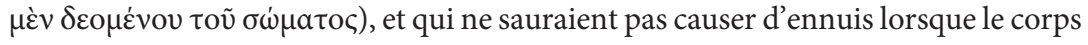
en a vraiment besoin. Quant à lui, il s'était manifestement si bien prémuni contre les plaisirs de l'amour, qu'il s'abstenait plus facilement des corps les plus beaux et

49. Cf. Mém. I 2, 1 et Dorion 2000, 9, n. 63.

50. Cf. Mém. II 1, 5 et II 2, 4 . 


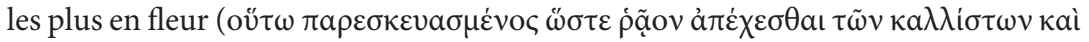

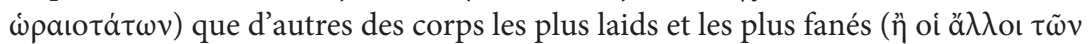

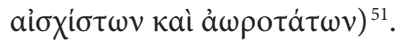

Je vois deux recoupements entre ces passages: premièrement, le conseil que Socrate adresse à ceux qui ne sont pas fermes ou endurcis à l'endroit des plaisirs d'Aphrodite

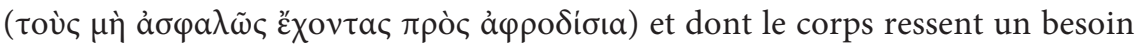

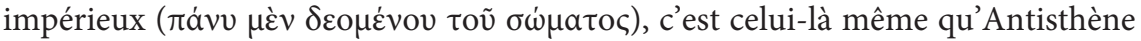
met en pratique lorsque son corps éprouve le besoin de rapports sexuels (

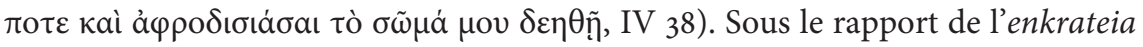
sexuelle, Antisthène apparaît donc nettement inférieur à Socrate; or, comme l'enkrateia est une condition de l'autarcie, il est par le fait même moins autarcique que Socrate. Deuxièmement, lorsque Xénophon ajoute que Socrate «avait plus de facilité à s'abstenir des corps les plus beaux et les plus dans la fleur de l'âge (oü $\omega$

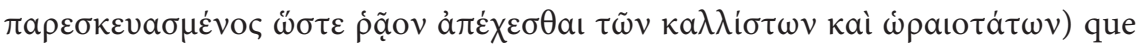

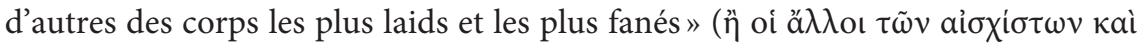

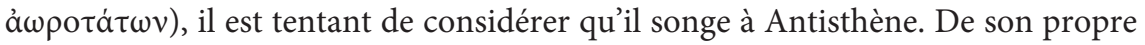
aveu, Antisthène se satisfait si bien des "premières venues » qu'il reçoit les caresses de femmes que personne d'autre n'aurait approchées (IV 38); et comme il ne fait rien pour résister au désir qu'il est prêt à satisfaire auprès de ces laiderons, il est très tentant de considérer que la pointe contenue à la fin de I 3,14 vise précisément Antisthène.

Si l'on prend en considération, d'une part les nombreux recoupements entre la position d'Antisthène et celle de Socrate, d'autre part les quelques écarts qui trahissent la distance qui sépare l'autarcie d'Antisthène de celle de Socrate, on peut raisonnablement conclure que Xénophon cherche à montrer qu'Antisthène est un authentique disciple de Socrate, qui a déjà beaucoup profité de son enseignement et de son exemple, mais qui n'est pas pour autant parvenu à égaler son maître. Enfin, on peut également observer que des sept discours présentés au chapitre IV, il y en a trois qui concernent l'argent (Callias, Charmide et Antisthène) et qu'un seul d'entre eux, celui d'Antisthène, exprime sur l'argent un point de vue authentiquement socratique.

\section{Hermogène (IV 46-49)}

À l'instar de Critobule et d'Antisthène, Hermogène est un personnage auquel Xénophon accorde un rôle beaucoup plus considérable que ne le fait Platon. Ainsi

51. Mém. I 3, 14 (trad. Dorion 2000). 
est-ce à lui que s'en remet Xénophon pour le récit du procès de Socrate ${ }^{52}$. Et à l'exemple de Critobule ${ }^{53}$ et d'Antisthène ${ }^{54}$, Hermogène ${ }^{55}$ est un des principaux personnages des entretiens sur l'amitié au livre II des Mémorables.

Hermogène est fier de compter les dieux au nombre de ses amis. Il s'est gagné leur amitié de la même façon que Cyrus l'a obtenue d'eux, à savoir en leur témoignant des égards et par des marques de piétée ${ }^{56}$. Il n'y a pas lieu de s'étonner de voir Socrate s'exclamer qu'il n'y a rien d'incroyable dans le discours d'Hermogène (cf. $\$ 49$ ), puisqu'il soutient lui-même ailleurs chacune des affirmations avancées par Hermogène sur la nature des dieux ${ }^{57}$. À la différence d'Antisthène, qui énonce quelques affirmations ayant pour effet de mettre en lumière son «retard» par rapport à Socrate, Hermogène n'avance rien dans son discours qui ne corresponde exactement à ce que Socrate affirme lui-même ailleurs chez Xénophon. Est-ce à dire qu'Hermogène est le parfait disciple de Socrate, celui qui serait parvenu à s'égaler au maître? Bien qu'il soit d'une piété irréprochable, Hermogène est sur un point moins favorisé des dieux que ne l'est Socrate, de sorte qu'il semble moins cher ( $\varphi$ i $\lambda \circ \varsigma)$ aux dieux, c'est-à-dire ami ( $\varphi$ i $\lambda \circ \varsigma)$ des dieux, que son maître. Il doit, en effet, avoir recours à la divination pour connaître ce que les dieux lui enjoignent ou lui interdisent de faire $(\$ 48)$, alors que Socrate peut s'en remettre au signe qui lui est particulier et par le truchement duquel les dieux lui révèlent directement, sans aucun intermédiaire, ce qu'il doit faire ou s'abstenir de faire. Au livre IV des Mémorables, après que Socrate lui a expliqué que la divination permet aux hommes de connaître à l'avance ce que les dieux leur réservent, Euthydème observe que Socrate a droit à un traitement plus amical ( $\varphi \iota \lambda \iota \kappa \omega ́ \tau \varepsilon \rho o v)$, puisque les dieux lui communiquent directement, par l'intermédiaire de son signe divin, ce qu'il doit faire ou s'abstenir de faire.

[Socrate] En outre, si nous sommes incapables de prévoir ce que le futur nous réserve d'utile, ils ( $s c$. les dieux) nous secondent dans cette tâche en révélant par

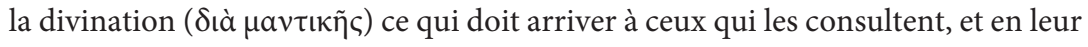

52. Cf. Apol. 2; Mém. IV 8, 4.

53. Cf. Mém. II 6.

54. Cf. Mém. II 5.

55. Cf. Mém. II 10.

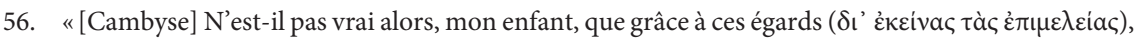
tu as plus de joie maintenant à aller prier les dieux, plus d'espoir d'obtenir d'eux ce dont tu as besoin, parce que tu as conscience de ne les avoir jamais négligés? - [Cyrus] Assurément, père, dit

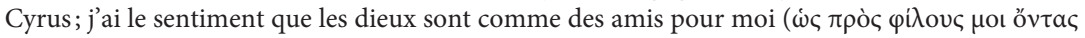

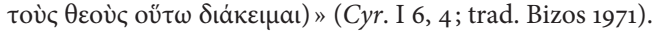

57. Voir, en Annexes à cette étude, le Tableau des correspondances et des divergences entre Socrate et ses compagnons. 
enseignant de quelle façon tirer des événements le meilleur parti. - [Euthydème] Mais toi, Socrate, reprit-il, il semble qu'ils te réservent un traitement encore plus amical ( $\varphi \iota \lambda เ \kappa \omega ́ \tau \varepsilon \rho o v)$ qu'aux autres hommes, puisque, sans même que tu les inter-

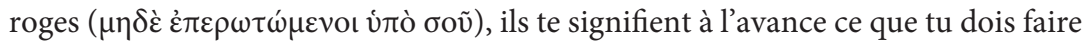
ou non ${ }^{58}$.

Tous les hommes peuvent faire appel à la divination, mais cela n'entraîne pas que les dieux traitent tous les hommes sur le même pied, c'est-à-dire qu' ils font à tous les hommes sans exception des révélations équivalentes sur leur avenir respectif. Il faut, en effet, mériter leur bienveillance pour que les dieux consentent à faire des révélations ${ }^{59}$. Comme Socrate a droit à un traitement encore plus amical ( $\left.\varphi \iota \lambda \iota \kappa \omega ́ \tau \varepsilon \rho o v\right)$, en ce sens que les dieux lui font des révélations sans même qu'il les interroge, il s'ensuit qu'il est le plus pieux des hommes et qu'il est encore plus qu'Hermogène traité en ami par les dieux.

La raison pour laquelle le discours d'Hermogène sur l'amitié des dieux fait immédiatement suite à celui d'Antisthène sur l'autarcie est peut-être la suivante: Antisthène enseigne l'art d'être dépourvu de besoins; or, l'autosuffisance est une des principales caractéristiques de la divinité ${ }^{60}$; il est donc naturel, vu sous cet angle, que les thèmes de la divinité et de la piété succèdent à celui de l'autarcie.

\section{Socrate (IV 56-64)}

Socrate est fier de son rôle d'entremetteur (mastropos). Le discours où il explique en quoi consiste son rôle d'entremetteur, et pourquoi il en est fier, illustre à merveille un des thèmes du Banquet, à savoir l'art de présenter en badinant des sujets sérieux. L'art de Socrate, tel qu'il est présenté en IV 56-64, est tout à fait sérieux ${ }^{61}$, mais Socrate l'introduit de telle façon que tout le monde éclate de rire (III 10). Et il semble qu'aucun autre convive ne réussisse aussi bien que Socrate dans cet art de la plaisanterie sérieuse, ou du sérieux badin.

Il est important de souligner que la compétence de Socrate ne se limite pas à l'art de l'entremetteur et qu'il pourrait également s'enorgueillir de ce qui fait la fierté d'Antisthène et celle d'Hermogène. Socrate n'est, en effet, pas moins riche qu'Antisthène et pas moins pieux qu'Hermogène. Il cumule donc, en plus de l'art de l'entremetteur, l'art de n'avoir besoin de rien et la piété qui lui permet d'obtenir l'amitié des dieux. On pourrait également compter au nombre de ses compétences

\footnotetext{
58. Mém. IV 3, 12 (trad. Dorion 2011b).

59. Cf. Mém. I 1, 9 ; Écon. XI 8; Cyr. I 6, 46.

60. Cf. Mém. I 6, 10.

61. Cf. Dorion 2009.
} 
le talent que Callias a tort de revendiquer. Socrate sait, en effet, comment il faut $s^{\prime} y$ prendre pour rendre les hommes justes ${ }^{62}$, alors que Callias s'imagine à tort que c'est grâce à son argent qu'il parviendra à les rendre tels.

\section{Conclusion}

Dans le Banquet de Platon, Socrate ne donne son accord à aucun des discours que les autres convives ont prononcés sur le thème de l'amour. Il y a certes des éléments de ces discours qu'il reprend à son compte ${ }^{63}$, mais il ne souscrit à aucun discours dans son intégralité. Chez Xénophon, les discours d'Hermogène et d'Antisthène pourraient très bien, à quelques détails près, être prononcés par Socrate. Il s'agit là d'une différence importante par rapport au Banquet de Platon et que l'on peut sans doute interpréter comme suit: en prêtant à des proches de Socrate des discours authentiquement socratiques, Xénophon montre que Socrate parvenait bel et bien à rendre ses compagnons meilleurs par la transmission de ses vertus. Le Socrate de Xénophon semble donc réussir à rendre ses compagnons vertueux et semblables à lui, alors que le Socrate de Platon en est apparemment incapable.

Chez Platon, l'ordre de succession des discours est fixé en fonction de la place que les convives occupent, c'est-à-dire qu'on trace un cercle en commençant par la droite $(177 \mathrm{~d})^{64}$. Il n'y a donc a priori aucune raison pour qu'il y ait progression entre les discours, puisque l'ordre de succession ne correspond pas au mérite, à la vertu ou à la « socraticité» des convives, mais tout simplement à leur emplacement. Cela dit, rien n'empêche qu'à l'ordre de succession selon l'emplacement corresponde également une réelle progression, auquel cas Platon aurait fait coïncider l'ordre de succession selon l'emplacement et la progression selon le mérite, mais il ne semble pas que ce soit le cas. Dans le Banquet de Xénophon, l'ordre de succession des discours correspond à un rapprochement progressif avec Socrate, c'est-à-dire que plus on progresse dans la succession des discours, plus on se rapproche des positions exprimées, défendues et illustrées par Socrate. De fait, les premiers discours sont prononcés par des personnages - Callias, Nikératos - qui n'appartiennent pas au cercle socratique, alors que les derniers - ceux d'Antisthène et d'Hermogène - sont non seulement prononcés par des proches de Socrate, mais ils sont également, à quelques détails près qui trahissent en fait la distance qui sépare malgré tout les

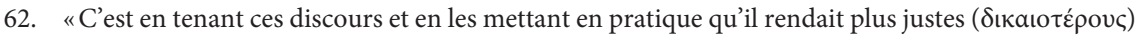
ceux qui le fréquentaient» (Mém. IV 4, 25 [trad. Dorion 2011b]).

63. Par exemple, Aristophane et Socrate affirment l'un et l'autre que l'objet de l'amour est ce qui nous est apparenté (cf. 192b et 205e); mais ils ne conçoivent pas de la même façon ce qui nous est apparenté.

64. L'ordre des éloges de l'Amour est donc le suivant: Phèdre (178a-18ob), Pausanias (18oc-185c), Éryximaque (185e-188e), Aristophane (189a-193d), Agathon (194e-197e), Socrate (198a-212c). 
disciples de leur maître, conformes et fidèles à l'enseignement de Socrate. Xénophon poursuit ainsi le programme apologétique qu'il formule dans les Mémorables et qui consiste à montrer que Socrate a été utile à ses compagnons et qu'il est réellement parvenu à les rendre vertueux $\left(\mathrm{I}_{3}, 1\right)$.

Dans son étude sur le Banquet de Xénophon, J.-C. Carrière isole Socrate de tous les autres personnages du dialogue, comme si Socrate n'avait rien en commun avec aucun de ces personnages:

Son Socrate [sc. de Xénophon] semble bien isolé au milieu des gentilshommes vaniteux, des pères La Vertu et des bouffons qui l'entourent, face à la sophistique de Callias, à la sotte érudition de Nikératos, au cynisme de Charmide, à une certaine niaiserie de Critobule, au moralisme et à la religiosité vides et brutaux d'Antisthène ou d'Hermogène ${ }^{65}$.

Pour les raisons qui ont été développées dans cette étude, je ne peux évidemment pas souscrire au jugement négatif que J.-C. Carrière porte sur Antisthène et Hermogène ${ }^{66}$, comme si ces derniers étaient aussi ridicules que Callias, Nikératos, Charmide et Critobule. Si la religiosité d'Hermogène est «vide», il faudrait nécessairement conclure, au vu de tous les recoupements entre sa piété et celle de Socrate, que la «religiosité» de Socrate l'est également ${ }^{67}$. De plus, si Xénophon ne parvenait pas à montrer que certains compagnons de Socrate sont réellement vertueux, il aurait par le fait même lamentablement failli à montrer que la compagnie de Socrate leur est profitable.

J'aimerais, en terminant, citer et commenter brièvement un passage tiré du Jean Santeuil de Marcel Proust. M. Beulier, le professeur de philosophie, fait découvrir à Jean la simplicité et la profondeur discrète d'un entretien rapporté par Xénophon dans les Mémorables (II 7):

Il sonna de nouveau Mariette, se fit donner les Mémorables de Xénophon et lut l'histoire de cette famille qui s'ennuyait, trouvait la vie mauvaise, vivait dans la division, et que Socrate rendit non seulement utile mais sage, heureuse et bonne, en la faisant travailler. Après les pages de Michelet la simplicité nue, la sécheresse de ce récit causèrent à Jean quelque déception. «Non, lui dit $\mathrm{M}$. Beulier, ce n'est pas moins bien, c'est autre chose, dame, il y a bien des choses, n'est-ce pas, l'Antiquité n'est pas le XIX ${ }^{e}$ siècle. Mais c'est aussi admirable. On n'écrira plus jamais ainsi. C'est tout à fait simple et pourtant tout est dit. C'est une époque où on ne développait

65. Carrière 1998, 270.

66. Voir aussi Carrière 1998, 256.

67. Cf. Huss 1999, 292: «Was X. dem Hermogenes hier in den Mund legt, ist X.s eigener traditioneller Religiosität und seiner verehrungsvollen Haltung gegenüber den traditionellen griechischen Göttern sehr verwandt». 
pas les idées, on les présentait ainsi sans les ouvrir, sans faire sortir tout ce qu'elles contenaient. Le duvet, la fraîcheur n'en étaient pas ôtés.» À la place où son maître avait semé un seul mot, Jean, qui le cultivait avec amour, trouvait au bout de quelque temps une idée florissante. Il trouva plus tard quand il le relut plus de charme qu'il n'aurait cru dans ce récit de Xénophon. Il y revint souvent dans la suite, et quand il avait dans sa chambre des amis intelligents et qui n'étaient point trop pressés, prenant involontairement par moments la voix chantante de M. Beulier il aimait bien le leur lire ${ }^{68}$.

C'est une belle leçon d'humilité que d'être ainsi conduit à reconnaître qu'un texte simple, en apparence banal, est en réalité beaucoup plus profond qu'on ne s'en doutait à la première lecture. Les Mémorables sont ainsi pour le narrateur de Jean Santeuil ce que François le Champi de George Sand est au narrateur de la Recherche $d u$ temps perdu $u^{69}$. Présenter des idées sans les ouvrir, sans les développer, sans faire sortir tout ce qu'elles contiennent, c'est également ce que fait Xénophon dans le Banquet, et il nous appartient à nous, lecteurs modernes, à nous, interprètes des écrits socratiques de Xénophon, d'ouvrir ces idées avec soin, de les développer avec patience et délicatesse, afin de révéler et de faire sortir tout ce qu'elles renferment. C'est à cet exercice de patience que je me suis appliqué humblement, en cherchant à mettre en lumière la «logique» tacite qui préside à la succession des discours dans le chapitre IV du Banquet.

Louis-André DORION

Université de Montréal

68. M. Proust, Jean Santeuil, Paris, Gallimard (Bibliothèque de la Pléiade; 228), 1971, p. 267.

69. M. Proust, À la recherche du temps perdu, Paris, Gallimard (Bibliothèque de la Pléiade; 100), 1954, vol. I, p. 41-43. 


\section{Références bibliographiques}

Bizos M. (1971), Xénophon. Cyropédie, t. I: Livres I-II, Paris, Les Belles Lettres (CUF; 204).

CARrière J.-C. (1998), «Socratisme, platonisme et comédie dans le Banquet de Xénophon ", in Le rire des Anciens, P. Hoffmann et M. Trédé (dir.), avec la collaboration de C. Auvray-Assayas, Paris, Presses de l’École normale supérieure (Études de littérature ancienne; 8), p. 243-271.

Chantraine P. (1949), Xénophon. Économique, P. Chantraine (éd. et trad.), Paris, Les Belles Lettres (CUF; 111).

Dorion L.-A. (2000), Xénophon. Mémorables, t. I: Introduction générale. Livre I, M. Bandini (éd.), L.-A. Dorion (trad.), Paris, Les Belles Lettres (CUF; 399).

Dorion L.-A. (2009), «Socrate entremetteur», Études platoniciennes, vol. VI: Socrate, vie privée, vie publique, p. 107-123; repris dans Dorion 2013, p. 347-368.

Dorion L.-A. (2011a), Xénophon. Mémorables, t. II-1: Livres II et III, M. Bandini (éd.), L.-A. Dorion (trad.), Paris, Les Belles Lettres (CUF; 477).

Dorion L.-A. (2011b), Xénophon. Mémorables, t. II-2: Livre IV, M. Bandini (éd.), L.-A. Dorion (trad.), Paris, Les Belles Lettres (CUF; 478).

Dorion L.-A. (2013), L'autre Socrate. Études sur les écrits socratiques de Xénophon, Paris, Les Belles Lettres (L'Âne d'or; 40).

Huss B. (1999), Xenophons Symposion. Ein Kommentar, Stuttgart - Leipzig, B.G. Teubner (Beiträge zur Altertumskunde; 125).

NaIls D. (2002), The people of Plato. A prosopography of Plato and other Socratics, Indianapolis - Cambridge, Hackett Publishing Co.

Ollier F. (1961), Xénophon. Banquet, F. Ollier (éd. et trad.), Paris, Les Belles Lettres (CUF; 153). 
ANNEXes

Tableau des correspondances et des divergences entre Socrate et ses compagnons ${ }^{70}$

\begin{tabular}{|c|c|c|c|c|c|}
\hline Sujet & Critobule & Charmide & Antisthène & Hermogène & $\begin{array}{l}\text { Socrate (ou } \\
\text { Xénophon) }\end{array}$ \\
\hline $\begin{array}{l}\text { 1. Incarne le } \\
\text { jeune homme } \\
\text { dépourvu } \\
\text { d'enkrateia }\end{array}$ & $\begin{array}{l}\text { IV 10-28; } \\
\text { Mém. I 3, } \\
\text { 8-13; II 6; } \\
\text { Écon. I } 16 \\
\text { - II } 8\end{array}$ & & & & \\
\hline $\begin{array}{l}\text { 2. Recherche } \\
\text { exclusive de la } \\
\text { beauté physique }\end{array}$ & $\begin{array}{l}\text { IV 10-13; } \\
\text { IV } 15-16 ; \\
\text { IV } 19\end{array}$ & & & & $\neq$ Mém. IV 1,2 \\
\hline $\begin{array}{l}\text { 3. Effet de } \\
\text { l'amour sur } \\
\text { l'aimé }\end{array}$ & $\begin{array}{l}\text { IV 13; } \\
\text { IV } 24\end{array}$ & & & & $\neq \mathrm{I} 10$ \\
\hline $\begin{array}{l}\text { 4. Le labeur } \\
\text { (ponos) n'est } \\
\text { pas nécessaire } \\
\text { pour obtenir } \\
\text { les biens: } \\
\text { la beauté suffit }\end{array}$ & IV 13 & & & & $\begin{array}{l}\neq \text { VIII } 32 \text {, VIII } 37 \text {, } \\
\text { VIII } 40 ; \\
\text { Mém. II } 1,20 ; \\
\text { II 1, 23-25; } \\
\text { II } 1,28 ; \text { II } 1,33-34\end{array}$ \\
\hline $\begin{array}{l}\text { 5. L'esclavage } \\
\text { amoureux }\end{array}$ & IV 14 & & & & $\neq$ Mém. I 3, 11 \\
\hline $\begin{array}{l}\text { 6. La beauté } \\
\text { favorise la } \\
\text { pudeur et la } \\
\text { maîtrise de soi } \\
\text { (enkrateia) }\end{array}$ & IV 15 & & & & $\begin{array}{l}\text { \# IV 26; I 8; } \\
\text { VIII 27; VIII } 33 \text {; } \\
\text { VIII } 35\end{array}$ \\
\hline $\begin{array}{l}\text { 7. La beauté } \\
\text { des stratèges }\end{array}$ & IV 16 & & & & $\neq$ Mém. III 1-7 \\
\hline $\begin{array}{l}\text { 8. Passer à } \\
\text { travers le feu }\end{array}$ & IV 16 & & & & $\neq$ Mém. I 3,9 \\
\hline $\begin{array}{l}\text { 9. L'utilité } \\
\text { de la beauté }\end{array}$ & IV 16 & & & & $\begin{array}{l}\text { ₹ IV 24; } \\
\text { Mém. I 5, } 4\end{array}$ \\
\hline
\end{tabular}

70. Ce tableau ne comprend donc pas les différents points d'opposition contenus dans les discours de Callias et de Nikératos. 
Ordre ET PRogression des discours AU CHAPITRE IV DU BANQUET...

\begin{tabular}{|c|c|c|c|c|}
\hline $\begin{array}{l}\text { 10. Obtenir } \\
\text { des faveurs } \\
\text { par persuasion }\end{array}$ & IV 18 & & & $\begin{array}{l}\text { ₹ VIII } 20 ; \\
\text { Mém. III } 11\end{array}$ \\
\hline $\begin{array}{l}\text { 11. Le corps est } \\
\text { plus efficace que } \\
\text { la parole pour } \\
\text { séduire }\end{array}$ & IV 18 & & & $\begin{array}{l}\text { = Mém. III 11; } \\
\text { IV } 2\end{array}$ \\
\hline $\begin{array}{l}\text { 12. La violence } \\
\text { de la passion } \\
\text { amoureuse }\end{array}$ & IV 23 & & & $\neq \mathrm{I} 10$ \\
\hline $\begin{array}{l}\text { 13. Le danger } \\
\text { du baiser }\end{array}$ & & & & $\begin{array}{l}\text { IV 25-26 } \\
=\text { Mém. I 3, 12; } \\
\text { II 6, 32-33 }\end{array}$ \\
\hline $\begin{array}{l}\text { 14. Modération } \\
\text { (sôphrosunê) } \\
\text { et «abstinence» }\end{array}$ & & & & $\begin{array}{l}\text { IV } 26 \\
=\text { Mém. I 3, } 8\end{array}$ \\
\hline $\begin{array}{l}\text { 15. La «morsure» } \\
\text { des beaux } \\
\text { garçons }\end{array}$ & & & & $\begin{array}{l}\text { IV } 28 \\
=\text { Mém. I 3, } 12\end{array}$ \\
\hline $\begin{array}{l}\text { 16. Être fier } \\
\text { de sa pauvreté }\end{array}$ & & III 9; IV 29 & & $\neq$ Écon. XI 3 \\
\hline $\begin{array}{l}\text { 17. Courtiser } \\
\text { les sycophantes }\end{array}$ & & IV 30 & & ₹ Mém. II 9 \\
\hline $\begin{array}{l}\text { 18. N'est pas en } \\
\text { mesure de faire } \\
\text { du mal à ses } \\
\text { ennemis }\end{array}$ & & IV 30 & & f Mém. II 6, 35 \\
\hline $\begin{array}{l}\text { 19. Menace ses } \\
\text { concitoyens }\end{array}$ & & IV 31 & & $\begin{array}{l}\neq \text { Mém. I 2, 59; } \\
\text { II } 9\end{array}$ \\
\hline $\begin{array}{l}20 . \text { Conception } \\
\text { relativiste de la } \\
\text { richesse et de la } \\
\text { pauvreté }\end{array}$ & & & IV $34-45$ & $\begin{array}{l}=\text { Mém. IV 2, } \\
37-39 ; \\
\text { Écon. II 2-10 }\end{array}$ \\
\hline $\begin{array}{l}\text { 21. Être fier } \\
\text { de sa richesse }\end{array}$ & & & IV 34 & = Écon. II 2 ; XI 3 \\
\hline
\end{tabular}


LOUIS-ANDRÉ DORION

\begin{tabular}{|c|c|c|}
\hline $\begin{array}{l}\text { 22. Posséder } \\
\text { peu de biens }\end{array}$ & $\begin{array}{l}\text { IV } 34 \\
(\beta \rho \alpha \chi \varepsilon \dot{\alpha} \alpha \\
\varepsilon \chi^{\prime}(\omega v)\end{array}$ & 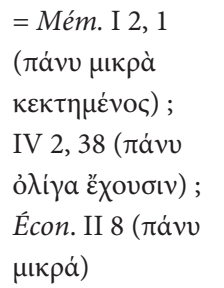 \\
\hline $\begin{array}{l}\text { 23. Tyrannie } \\
\text { et injustice }\end{array}$ & IV 36 & $\begin{array}{l}=\text { Mém. IV 2, } \\
38-39\end{array}$ \\
\hline $\begin{array}{l}\text { 24. Avoir } \\
\text { du superflu }\end{array}$ & $\begin{array}{l}\text { IV } 37 \\
(\pi \varepsilon \rho i \varepsilon \sigma \tau i \\
\mu о \mathrm{~s})\end{array}$ & $\begin{array}{l}=\text { Écon. II } 10 \\
(\pi \varepsilon \rho ı v \sigma i a)\end{array}$ \\
\hline $\begin{array}{l}25 . \text { Le plaisir } \\
\text { sexuel }\end{array}$ & IV 38 & $\neq$ Mém. I 3, 14-15 \\
\hline $\begin{array}{l}\text { 26. Le plaisir } \\
\text { du sommeil }\end{array}$ & IV 38 & $\neq$ Mém. I 6, 8 \\
\hline $\begin{array}{l}\text { 27. Travail } \\
\text { et subsistance }\end{array}$ & IV 40 & $\neq$ Mém. I 3, 5 \\
\hline $\begin{array}{l}\text { 28. Se régaler de } \\
\text { son «âme» }\end{array}$ & IV 41 & $\begin{array}{l}=\text { Apol. } 18 ; \\
\text { Mém. I 3, } 5\end{array}$ \\
\hline $\begin{array}{l}\text { 29. Plaisir } \\
\text { simple différé } v s \text {. } \\
\text { plaisir coûteux } \\
\text { immédiat }\end{array}$ & IV 41 & $\begin{array}{l}=\text { Mém. I 6, 5; } \\
\text { II } 1,30\end{array}$ \\
\hline $\begin{array}{l}\text { 30. Vin de } \\
\text { Thasos }\end{array}$ & IV 41 & $\begin{array}{l}=\text { Mém. I 6, 5; } \\
\text { II } 1,30 ; \text { IV 5, } 9\end{array}$ \\
\hline $\begin{array}{l}\text { 31. Frugalité } \\
\text { (euteleia) }\end{array}$ & IV 42 & $\begin{array}{l}=\text { Mém. I 3, 5; } \\
\text { III 13, 2; Apol. } 25\end{array}$ \\
\hline $\begin{array}{l}\text { 32. Contente- } \\
\text { ment et justice }\end{array}$ & IV 42 & $\begin{array}{l}=\text { Mém. I 2, } 1 \text { sq.; } \\
\text { IV 2, 38; Apol. } 16\end{array}$ \\
\hline $\begin{array}{l}\text { 33. Richesse } \\
\text { et générosité } \\
\text { (aphtonia) }\end{array}$ & IV 43 & $\begin{array}{l}=\text { Mém. I 2, 60; } \\
\text { I } 6,14\end{array}$ \\
\hline $\begin{array}{l}\text { 34. Le loisir } \\
(\text { skholê })\end{array}$ & IV 44 & $\begin{array}{l}=\text { Mém. I 6, 9; } \\
\text { III } 11,16\end{array}$ \\
\hline $\begin{array}{l}\text { 35. Fréquentation } \\
\text { de ceux qui } \\
\text { plaisent }\end{array}$ & IV 44 & $=$ Mém. I 2, 39 \\
\hline
\end{tabular}


Ordre ET PRogression des discours AU CHAPITRE IV DU BANQUET...

\begin{tabular}{|c|c|c|c|}
\hline $\begin{array}{l}\text { 36. Absence } \\
\text { de besoins } \\
\text { et autarcie }\end{array}$ & IV 45 & & $\begin{array}{l}=\text { Mém. I } 2,1 ; \\
\text { I } 2,14 ; \text { I } 2,60 ; \\
\text { I } 6,10 ; \text { IV } 7,1 ; \\
\text { IV } 8,11\end{array}$ \\
\hline $\begin{array}{l}\text { 37. Omniscience } \\
\text { des dieux }\end{array}$ & & IV 47 et 48 & $\begin{array}{l}=\text { Mém. I 1, 19; } \\
\text { I 4, 17-18 }\end{array}$ \\
\hline $\begin{array}{l}\text { 38. Pratique } \\
\text { universelle } \\
\text { de la divination }\end{array}$ & & IV 47 & = Mém. I 4, 15-16 \\
\hline $\begin{array}{l}\text { 39. Les dieux } \\
\text { peuvent nous } \\
\text { faire du bien } \\
\text { ou du mal }\end{array}$ & & IV 47 & = Mém. I 4, 16 \\
\hline $\begin{array}{l}\text { 40. Les dieux } \\
\text { font signe aux } \\
\text { hommes de leur } \\
\text { choix }\end{array}$ & & IV 48 & $\begin{array}{l}=\text { Mém. I 1, 3; } \\
\text { I 1, 19; Apol. } 13\end{array}$ \\
\hline $\begin{array}{l}\text { 41. Les dieux } \\
\text { se soucient } \\
\text { des hommes } \\
\text { (epimeleia) }\end{array}$ & & IV 46 et 48 & $\begin{array}{l}=\text { Mém. I } 4 \text { 13; } \\
14 ; \text { IV } 3,3 ; 12\end{array}$ \\
\hline $\begin{array}{l}\text { 42. Les dieux } \\
\text { connaissent } \\
\text { l'issue des } \\
\text { actions }\end{array}$ & & IV 48 & $=$ Mém. I 1, 7-9 \\
\hline $\begin{array}{l}\text { 43. Il faut } \\
\text { obéir aux } \\
\text { signes divins }\end{array}$ & & IV 48 & $=$ Mém. I 1, 4 \\
\hline $\begin{array}{l}\text { 44. On peut } \\
\text { obtenir l'amitié } \\
\text { des dieux }\end{array}$ & & IV 49 & $\begin{array}{l}=\text { Mém. II 1, 33; } \\
\text { IV 3, 12; } \\
\text { Cyrop. I 6, } 4\end{array}$ \\
\hline $\begin{array}{l}\text { 45. L'amitié } \\
\text { s'obtient par } \\
\text { l'éloge }\end{array}$ & & IV 49 & $\begin{array}{l}=\text { Mém. II 6, 9-13; } \\
\text { II 6, 33-38 }\end{array}$ \\
\hline $\begin{array}{l}\text { 46. Le culte } \\
\text { des dieux est } \\
\text { peu coûteux }\end{array}$ & & IV 49 & $\begin{array}{l}=\text { Mém. I 3, 3; } \\
\text { IV 3, 16-17 }\end{array}$ \\
\hline
\end{tabular}

